\title{
Caracterización en salud oral y hábitos de higiene oral en pacientes con enfermedad renal crónica
}

\author{
Diagnosis of oral health and oral hygiene habits in \\ patients with chronic kidney disease
}

\author{
Eliana E. Muñoz, César Augusto Restrepo, José Arnoby Chacón • \\ Manizales (Colombia)
}

\section{Resumen}

Objetivo: determinar el estado de salud oral de pacientes con enfermedad renal crónica atendidos en un centro de terapia renal y su relación con marcadores nutricionales, inflamatorios y dosis de eritropoyetina.

Pacientes: todos los pacientes con enfermedad renal crónica atendidos en RTS sucursal Caldas en programas predialítico, postrasplante renal, hemodiálisis y diálisis peritoneal, con más de tres meses ininterrumpidos de asistencia en uno de esos programas, haber aceptado su participación en el estudio, contar con los paraclínicos necesarios para el estudio y registro de dosis administrada de eritropoyetina.

Métodos: trescientos treinta y seis pacientes cumplieron con los criterios de inclusión: 24 (7\%) en prediálisis, 164 (49\%) en hemodiálisis, 114 (34\%) en diálisis peritoneal y 34 (10\%) en postrasplante renal. Se establecieron los datos demográficos y se evaluaron los índices de placa (higiene oral), salud periodontal, experiencia en caries COP-D (cariados, obturados, perdidos - diente), y diagnóstico estomatológico. También resultados de exámenes paraclínicos indicadores de estado inflamatorio tales como albúmina, ferritina y porcentaje de saturación de transferrina, además la dosis administrada de eritropoyetina.

Resultados: la población de pacientes en prediálisis y postrasplante renal no arrojó datos suficientes para ser incluida en el análisis estadístico. De los pacientes en diálisis $63 \%$ presentaban buena higiene oral, $33 \%$ regular, y sólo $3 \%$ tenían mala higiene oral, a pesar de ello la prevalencia de enfermedad periodontal fue alta afectando a $75 \%$ de los pacientes llegándose a presentar gingivitis severa en 35-38\% de los pacientes. Por el índice COP-D se observó que la prevalencia en caries fue alta. Por lo disperso de los resultados de laboratorio de marcadores inflamatorios no fue posible determinar correlación alguna entre ellos y salud periodontal o dosis de eritropoyetina.

Conclusiones: la higiene oral de los pacientes en diálisis es satisfactoria, pero a pesar de ello presentan alta prevalencia de enfermedad periodontal y caries, ello sugiere que factores relacionados con el medio urémico, modificaciones en el PH y flujo salival favorecen su aparición. La evaluación de estos parámetros en estudios posteriores podría ofrecer resultados más concluyentes, al igual que la utilización de marcadores inflamatorios más sensibles. La evaluación precoz de todos los pacientes con ERC por odontología es prioritaria para evitar la aparición de enfermedad periodontal. (Acta Med Colomb 2010; 36: 173-180)

Palabras clave: enfermedad renal crónica, enfermedad periodontal, salud oral.

\section{Abstract}

Objective: to establish the oral health condition of patients with chronic kidney disease (CKD) who received care in a center for renal therapy, as well as its relationship with nutritional and inflammatory markers and doses of erythropoietin received.

Patients: patients with chronic kidney disease treated at RTS Caldas, in the following programs: predialysis, post-renal transplant, hemodialysis, and peritoneal dialysis. All these patients had attended any of these programs uninterruptedLy for at least three months; they agreed to participate in the study and had the necessary laboratory studies, as well as registered doses of erythropoietin.
Dra. Eliana E. Muñoz López: Estomatóloga y Cirujana Oral. Profesor Asistente, Grupo INSAO, Universidad Autónoma de Manizales. Hospital Infantil Universitario de Manizales; Dr. César Augusto Restrepo Valencia: Internista-Nefrólogo. Profesor Asociado Universidad de Caldas, Director Cientifico RTS Sucursal Caldas; Dr. José Arnoby Chacón Cardona: MSc, Microbiología Médica. Profesor Titular Universidad de Caldas. Manizales (Colombia).

Correspondencia. Dr. César A. Restrepo V. E-mail: caugustorv@une.net.co Recibido: 27/VI/2011 Aceptado: 06/X/2011 
Methods: three hundred thirty-six patients met the inclusion criteria: 24 (7\%) in predialysis, 164 (49\%) in hemodialysis, 114 (34\%) in PD and 34 (10\%) in post-renal transplant. The following demographic data were established and assessed: Silness and Loe plaque indices (oral Health) and Loe and Silness gingival indices, caries experience in Decayed-Missing-Filled Teeth Index (DMFT) and stomatologic diagnosis. Additionally, laboratory reports were recorded indicating the inflammation state of the patients: albumin, ferritin, transferrin saturation and erythropoietin dosage.

Results: pre-dialysis and post-renal transplant populations did not give sufficient information to be included in the statistical analysis. The following information was obtained from dialysis patients: $63 \%$ had good oral hygiene, $33 \%$ fair, and only $3 \%$ had poor oral hygiene. The prevalence of periodontal disease was high, affecting $75 \%$ of patients. Severe gingivitis was present in $35-38 \%$ of patients. The DMFT index showed a high prevalence of caries. No relationship was found between laboratory results for inflammatory markers, periodontal health, and doses of erythropoietin.

Conclusions: the oral hygiene of dialysis patients is satisfactory. However, they have a high prevalence of periodontal disease and tooth decay. This suggests that factors related to the uremic medium, $\mathrm{pH}$ and salivary flow favor its appearance. The evaluation of these parameters in future studies could provide more conclusive results, as well as the use of more sensitive markers of inflammation. Early evaluation provided by dental professionals is a priority for all patients with CKD in order to prevent the occurrence of periodontal disease. (Acta Med Colomb 2010; 36: 173-180)

Keywords: chronic kidney disease, periodontal disease, oral health.

\section{Introducción}

Los pacientes con enfermedad renal crónica (ERC) presentan con frecuencia alteraciones en los hábitos de salud oral que predisponen a mala higiene bucal, y cambios en los mecanismos protectores de la cavidad oral, lo que favorece la aparición de complicaciones tales como candidiasis, queilitis angular, lengua saburral, gingivitis, periodontitis, destrucción periodontal severa, placa bacteriana, cálculo dental, movilidad dentaria y pérdida prematura de dientes. En pacientes con ERC en todos sus estadios se ha detectado una frecuencia alta de enfermedad periodontal, siendo mayor en pacientes en terapia hemodialítica en quienes afecta al $50 \%$ de la población (1).

Existe asociación demostrada entre enfermedad periodontal e inflamación sistémica, aspecto que podría sumarse a los factores clásicos asociados a complicaciones cardiovasculares en estos pacientes (2).

En el medio colombiano existen informes de la prevalencia de enfermedad periodontal en la población general que según la Tercera Encuesta de Salud Bucal (ENSAB III), comunicó para todas las formas de enfermedad periodontal (gingivitis y periodontitis) una prevalencia del $50.2 \%$, de esta cifra $17.7 \%$ de los casos corresponden a periodontitis de moderada a severa (3), pero faltan estudios que indiquen el grado de afectación de los pacientes con alguna forma de compromiso renal.

En el presente trabajo se desea caracterizar los hábitos de higiene oral y el estado de salud periodontal en pacientes con ERC que asisten a los programas de prediálisis, hemodiálisis, diálisis peritoneal y postrasplante renal en una unidad de terapia renal de la ciudad de Manizales, Caldas, Colombia, Sur América. Además se desea establecer si existe alguna correlación entre esos hábitos y diversas variables clínicas.

\section{Material y métodos}

Fueron considerados candidatos para participar en el estudio todos los pacientes que asistieron a RTS Sucursal Caldas en Manizales, Caldas, Colombia, Sur América entre los meses de septiembre del año 2009 a marzo de 2010, con diagnóstico de enfermedad renal crónica en atención predialítica (tasa de filtración glomerular menor a $30 \mathrm{ml} / \mathrm{minuto}$ y mayor a $15 \mathrm{ml} /$ minuto), postrasplante renal, hemodiálisis crónica o diálisis peritoneal, que llevaran más de tres meses de atención ininterrumpidos en este centro médico, y que firmaran el consentimiento informado para su participación. Fueron criterios de exclusión: presencia de neoplasia diseminada, cirrosis hepática, infección aguda de la cavidad oral o de las glándulas salivales, incapacidad de lograr una apertura bucal suficiente y el uso regular de anticoagulantes (excepto la heparina utilizada durante la terapia dialítica).

El estudio fue aprobado por el Comité de Investigaciones del Hospital Infantil Universitario de Manizales y el Grupo INSAO de la Universidad Autónoma de Manizales.

Luego de obtener el consentimiento, se realizó una encuesta al paciente en la cual se obtuvieron datos relacionados con aspectos demográficos, y por revisión de la historia clínica se tomaron los datos clínicos y de laboratorio, correspondientes al mismo mes de la evaluación de la salud oral. Se consideraron valores normales de albúmina entre 3.5-5 $\mathrm{gr} / \mathrm{dL}$, ferritina entre $100-500 \mathrm{mg} / \mathrm{dL}$ y $\%$ de saturación de transferrina mayor a 20\%. La condición de inflamación se estableció si se presentaba simultáneamente los siguientes valores de laboratorio: albúmina menor a $3.5 \mathrm{gr} / \mathrm{dL}$ en presencia de una nPCR (tasa catabólica proteica normalizada) mayor a 1.0 , ferritina mayor a $500 \mathrm{mg} / \mathrm{dL}$ y saturación de transferrina menor a $20 \%$. Estos marcadores se utilizaron puesto que se ha demostrado disminución en la síntesis de 
albúmina y transferrina en estados inflamatorios (marcadores inversos de fase aguda), y elevación en los niveles séricos de ferritina (4). No se utilizó la proteína $C$ reactiva puesto que a la fecha sólo la variedad altamente sensible es la recomendada (5), y su determinación es muy costosa en nuestro medio, al igual que la prealbúmina $(6,7)$. Se valoró además la dosis de eritropoyetina que recibían los pacientes para establecer la concordancia entre el estado inflamatorio (medido por índices de higiene oral, salud periodontal y resultados de laboratorio) y dosis utilizada, dividiendo los pacientes en dosis normal menor a 6000 UI por semana, $y$ alta mayor a 6000 UI por semana.

El examen de la cavidad oral fue realizado por dos odontólogas especialistas en estomatología y cirugía oral.El examen clínico intraoral de los pacientes en hemodiálisis se practicó directamente en el sillón de tratamiento minutos antes de iniciar su terapia dialítica.Los pacientes en prediálisis, diálisis peritoneal y postrasplante renal se evaluaron en un consultorio el mismo día del control médico de nefrología. El examen oral se hizo mediante secado con aire, inspección con bajalenguas con lámpara de luz artificial y espejo bucal.

Para el diagnóstico estomatológico se utilizaron las siguientes categorías: 1) "Sano": adecuada humectación en la mucosa oral, lengua con papilas de anatomía normal, encía libre, adherida, con coloración y estructura adecuadas. 2) "Lengua fisurada": fisuras sobre la superficie dorsal de la lengua que varían en tamaño y profundidad. 3) "Candidiasis" lengua roja, lisa, brillante, cubierta de placas blanquecinas y dolorosa o afectación de las comisuras bucales en forma de placas triangulares. (En los pacientes con sospecha clínica se tomó un frotis para examen directo confirmatorio). 4) "Pérdida de dimensión vertical" en pacientes que presentaron pérdida de estructuras dentales totales y uso sólo de prótesis total superior. 5) "Hiperplasia" por presencia de sobre crecimiento del tejido conectivo fibroso colágeno, caracterizado clínicamente por presentar un "tejido fuerte" limitado a una zona o involucrando varias regiones, condiciones que lo distinguen de la inflamación aguda. 6) "Xerostomía" por mucosa seca, y 7) "Lengua saburral" al detectarse sobre el dorso de la lengua una capa blanca de placa bacteriana.

Para la clasificación de la higiene oral se utilizó el índice de placa de acuerdo con Silness J y Loe H (8). Se evaluaron las cuatro superficies del último molar de cada cuadrante y los dientes 11,23 y 44 . Los valores fueron: $0=$ no hay placa en el área gingival, 1 = capa adherida al margen gingival libre y el área adyacente del diente, 2 = acúmulo moderado de depósitos blandos dentro de la bolsa gingival y en el margen gingival o adyacente a la superficie dentaria o ambos la cual puede verse a simple vista y $3=$ abundancia de materia blanda dentro de la bolsa gingival o en el margen gingival adyacente a la superficie dentaria o en ambos. De cada diente se tomaron los puntajes de cada cara y se sumaron. Este total se dividió entre 4 que es el número de superficies evaluadas en cada diente; se obtuvo así el promedio de cada diente, sumándose todos los promedios de cada diente y dividiéndose por el número de dientes evaluados para obtener finalmente el índice registrado. Las categorías anotadas para la higiene oral son: "Buena" entre 0 y 1 ; "Regular" entre 1.1 y 2 ; "Mala higiene oral" entre 2.1 y 3 . Para edéntulos no aplicó.

La salud periodontal se evaluó mediante el índice de Loe H y Silness J (9). Se evaluaron las cuatro superficies del último molar de cada cuadrante, además los dientes 11,23 y 44. Los valores fueron: 0 = encía normal, $1=$ inflamación leve: ligero cambio de color, edema leve, sin hemorragia a la palpación; 2 = inflamación moderada: color rojo, edema y aspecto brillante, hemorragia a la palpación; 3 = inflamación grave: marcado color rojo y edema, ulceraciones, tendencia a la hemorragia espontánea. De cada diente se tomaron los puntajes de cada cara y se sumaron, este total se dividió entre 4 que son el número de superficies evaluadas en cada diente; obteniéndose así el promedio de cada diente, sumándose todos los promedios de cada diente y dividiéndose por el número de dientes evaluados para obtener el índice registrado. Las categorías anotadas para la salud periodontal son: "normal" = 0 ; gingivitis "leve" entre $0.1-1$; gingivitis "moderada" entre 1.1-2; gingivitis "severa" entre 2.1-3.

La experiencia de caries se midió con el índice COP-D (cariados, obturados, perdidos - Diente ) el cual se obtiene de la sumatoria de los dientes permanentes cariados, perdidos y obturados, incluidas las extracciones indicadas. El resultado se dividió entre el total de dientes examinados. Se consideraron sólo 28 dientes, puesto que las cordales no se tienen en cuenta por ser dientes con exodoncia indicada dada su mal posición o retardo en la erupción en la generalidad de los pacientes. Según la OMS (10), el índice COP-D tiene 5 niveles así: a) "muy bajo": 0.0-1.1; b) "bajo": 1.2-2.6; c) "intermedio": 2.7$4.4 ;$ d) "alto": $4.5-6.5 ;$ e) "muy alto": 6.6 y más.

El análisis de las variables cualitativas se realizó mediante proporciones y de las cuantitativas a través de promedios y desviación estándar. Una p $<0.05$ fue considerada estadísticamente significativa. Para la recolección y análisis de la información se utilizó el programa SSPS Versión 11.

\section{Resultados}

Se evaluaron 336 pacientes en el periodo establecido, cuyas características demográficas se presentan en la Tabla 1. Veinticuatro (7.1\%) pacientes estaban en prediálisis, 34 (10.1\%) postrasplante renal, 164 (48.8\%) en hemodiálisis y $114(33.9 \%)$ en diálisis peritoneal.

El índice de placa bacteriana de Silness J. y Loe H. se aplicó a 209 (62\%) de 336 pacientes, los 127 (38\%) restantes no evaluados usaban prótesis total o eran edentados. Los resultados de higiene oral fueron: buena en 144 de $209(68 \%)$, regular en $58(27 \%)$ y mala en siete (3.34\%). En el análisis por tipo de tratamiento se encontró que en prediálisis sólo tres pacientes de 24 fueron evaluables; dos (66.6\%) presentaron buena higiene oral. De 132 pacientes evaluables en hemodiálisis, 84 (63.63\%) presentaban buena higiene oral, $44(33.33 \%)$ regular higiene y cuatro (3.03\%) mala higiene oral. De 63 pacientes que estaban en diálisis 
peritoneal, $46(68.2 \%)$ presentaban buena higiene oral, 13 $(20.63 \%)$ regular higiene y tres $(4.76 \%)$ presentaron mala higiene. De los 12 pacientes del grupo de trasplantados, todos presentaron buena higiene oral (Tabla 2).

El índice de Loe $\mathrm{H}$ y Silness $\mathrm{J}$ de salud periodontal también se aplicó a 209 de 336 pacientes, los 127 (38\%) restantes no evaluables usaban prótesis total o eran edentados. Se obtuvieron los siguientes resultados: normal en 52 de 209 (24.88\%), anormal en 157 (75.11\%) distribuidos así: gingivitis leve en 47/209 (22.48\%), moderada en 42/209 (20.09\%) y severa en 68/209 (35.53\%). En el análisis por grupos de tratamiento se encontró que de los tres pacientes evaluables que estaba en prediálisis dos (66.6\%) estaban sanos y uno $(33.33 \%)$ presentó gingivitis severa. De 132 pacientes evaluables en hemodiálisis, 28 (21.21\%) se detectaron sanos y $104(78.78 \%)$ presentaron algún grado de gingivitis distribuida así: 26 (19.69\%) con gingivitis leve, $27(20.45 \%)$ gingivitis moderada y $51(38.63 \%)$ presentaron gingivitis severa. Entre los 63 pacientes evaluables en diálisis peritoneal $16(25.39 \%)$ se encontraron sanos, y 47
(74.60\%) tenían algún grado de gingivitis distribuida así: $17(26.90 \%)$ con gingivitis leve, $14(22.22 \%)$ gingivitis moderada y $16(25.39 \%)$ presentaron gingivitis severa. De los 11 pacientes del grupo de trasplantados, seis $(54.54 \%)$ se presentaron sanos, cuatro $(36.36 \%)$ tenían gingivitis leve, uno $(9.09 \%)$ gingivitis moderada (Tabla 2$)$.

La experiencia de caries identificada por medio del índice COP-D fue encontrado así: muy bajo en 106 (32\%) pacientes; bajo en un paciente $(1 \%)$; intermedio en seis pacientes (2\%); alto en cinco pacientes $(1 \%)$ y muy alto en 218 pacientes (64\%) (Tabla 2 ).

En el diagnóstico estomatológico, 53 (16\%) pacientes se observaron sanos, 130 (39\%) con lengua saburral, 54 (16\%) con lengua fisurada y en $22(6.54 \%)$ se detectó candidiasis dentro de este grupo; en $72(21.4 \%)$ pacientes se observó pérdida de la dimensión vertical, en 27 (8\%) otros diagnósticos. La distribución del diagnóstico estomatológico por tipo de atención se indica en la Tabla 2.

En relación con marcadores inflamatorios se detectó baja saturación de transferrina (menor a 20\%) en uno de los 24

Tabla 1. Características demográficas de pacientes en terapia renal evaluados en su salud oral.

\begin{tabular}{|c|c|c|c|c|c|c|c|c|c|c|}
\hline \multirow{2}{*}{$\begin{array}{l}\text { Características } \\
\text { demográficas }\end{array}$} & \multicolumn{2}{|c|}{ Prediálisis } & \multicolumn{2}{|c|}{ Hemodiálisis } & \multicolumn{2}{|c|}{ Diálisis peritoneal } & \multicolumn{2}{|c|}{ Trasplante renal } & \multicolumn{2}{|c|}{ Total } \\
\hline & $\mathbf{n}$ & $(\%)$ & $\mathbf{n}$ & $(\%)$ & $\mathbf{n}$ & $(\%)$ & $\mathbf{n}$ & $(\%)$ & $\mathbf{n}$ & $(\%)$ \\
\hline GÉNERO & & & & & & & & & & \\
\hline Masculino & 14 & 4 & 105 & 31 & 64 & 19 & 23 & 7 & 206 & 61.3 \\
\hline Femenino & 10 & 3 & 59 & 18 & 50 & 15 & 11 & 3 & 130 & 38.6 \\
\hline Total & 24 & 7 & 164 & 49 & 114 & 34 & 34 & 10 & 336 & 100 \\
\hline GRUPO ETÁREO & & & & & & & & & & \\
\hline$<30$ & 0 & 0 & 10 & 3 & 3 & 1 & 6 & 2 & 19 & 6 \\
\hline 30 - 49 años & 1 & 0.3 & 36 & 11 & 32 & 10 & 17 & 5 & 86 & 26 \\
\hline $50-69$ años & 4 & 1 & 70 & 21 & 51 & 15 & 11 & 3 & 136 & 40 \\
\hline 70 y más & 19 & 6 & 48 & 14 & 28 & 8 & 0 & 0 & 95 & 28 \\
\hline Total & 24 & 7 & 164 & 49 & 114 & 34 & 34 & 10 & 336 & 100 \\
\hline ESTADO CIVIL & & & & & & & & & & \\
\hline Soltero & 1 & 1 & 43 & 12 & 19 & 5 & 12 & 4 & 75 & 22 \\
\hline Casado & 12 & 4 & 78 & 23 & 68 & 21 & 18 & 5 & 176 & 53 \\
\hline Separado & 0 & 0 & 9 & 2 & 5 & 1 & 1 & 1 & 15 & 4 \\
\hline Viudo & 9 & 2 & 23 & 6 & 13 & 4 & 1 & 1 & 46 & 13 \\
\hline Unión libre & 2 & 1 & 11 & 3 & 9 & 3 & 2 & 1 & 24 & 8 \\
\hline Total & 24 & 7 & 164 & 49 & 114 & 34 & 34 & 10 & 336 & 100 \\
\hline ESTRATO SOCIAL & & & & & & & & & & \\
\hline Estrato 1 & 1 & 0.5 & 6 & 2 & 29 & 8 & 1 & 0.5 & 37 & 11 \\
\hline Estrato 2 & 12 & 4 & 38 & 11 & 35 & 10 & 9 & 3 & 94 & 28 \\
\hline Estrato 3 & 6 & 2 & 91 & 27 & 38 & 11 & 20 & 6 & 155 & 46 \\
\hline Estrato 4 & 3 & 1 & 19 & 5 & 7 & 2 & 3 & 1 & 32 & 9 \\
\hline Estrato 5 & 1 & 0.5 & 3 & 1 & 4 & 1 & 1 & 0.5 & 9 & 3 \\
\hline Estrato 6 & 1 & 0.5 & 7 & 2 & 1 & 0.5 & 0 & 0 & 9 & 3 \\
\hline Total & 24 & 7 & 164 & 49 & 114 & 34 & 34 & 10 & 336 & 100 \\
\hline OCUPACIÓN & & & & & & & & & & \\
\hline Desempleado & 1 & 0.5 & 32 & 9.5 & 20 & 6 & 3 & 1 & 56 & 17 \\
\hline Empleado & 3 & 1 & 38 & 11 & 25 & 8 & 14 & 4 & 80 & 24 \\
\hline Estudiante & 0 & 0 & 3 & 0.5 & 0 & 0 & 2 & 0.5 & 5 & 1 \\
\hline Pensionado & 13 & 4 & 52 & 16 & 27 & 8 & 11 & 3 & 103 & 31 \\
\hline Ama de casa & 7 & 2 & 39 & 12 & 42 & 12 & 4 & 1 & 92 & 27 \\
\hline Total & 24 & 7 & 164 & 49 & 114 & 34 & 34 & 10 & 336 & 100 \\
\hline EDUCACIÓN & & & & & & & & & & \\
\hline Ninguna & 2 & 1 & 17 & 4 & 6 & 2 & 0 & 0 & 25 & 7 \\
\hline Primaria & 16 & 5 & 88 & 26 & 82 & 24 & 10 & 3 & 196 & 58 \\
\hline Secundaria & 4 & 1 & 48 & 14 & 15 & 5 & 16 & 5 & 83 & 25 \\
\hline Superior & 2 & 1 & 10 & 3 & 9 & 3 & 8 & 2 & 29 & 9 \\
\hline Posgrado & 0 & 0 & 1 & 0.2 & 2 & 1 & 0 & 0 & 3 & 1 \\
\hline Total & 24 & 7 & 164 & 49 & 114 & 34 & 34 & 10 & 336 & 100 \\
\hline
\end{tabular}


Tabla 2. Análisis de variables clínicas por grupos de pacientes en terapia renal evaluados en su salud oral.

\begin{tabular}{|c|c|c|c|c|c|c|c|c|c|c|}
\hline \multirow{2}{*}{ Variables Clínicas } & \multicolumn{2}{|c|}{ Prediálisis } & \multicolumn{2}{|c|}{ Hemodiálisis } & \multicolumn{2}{|c|}{ Diálisis peritoneal } & \multicolumn{2}{|c|}{ Trasplante renal } & \multicolumn{2}{|c|}{ Total } \\
\hline & $\mathrm{n}$ & $(\%)$ & $\mathbf{n}$ & $(\%)$ & $\mathbf{n}$ & (\%) & $\mathbf{n}$ & $(\%)$ & $\mathbf{n}$ & $(\%)$ \\
\hline $\begin{array}{l}\text { ETIOLOGÍA } \\
\text { Nefropatía hipertensiva } \\
\text { Nefropatía diabética } \\
\text { Etiología desconocida } \\
\text { Nefropatía obstructiva } \\
\text { Glomerulonefritis } \\
\text { Otras causas } \\
\text { Total }\end{array}$ & $\begin{array}{c}8 \\
4 \\
3 \\
5 \\
1 \\
3 \\
\mathbf{2 4}\end{array}$ & $\begin{array}{l}3 \\
1 \\
1 \\
2 \\
1 \\
1 \\
7\end{array}$ & $\begin{array}{c}31 \\
38 \\
39 \\
24 \\
12 \\
20 \\
\mathbf{1 6 4}\end{array}$ & $\begin{array}{c}9 \\
11 \\
11 \\
6 \\
4 \\
6 \\
49\end{array}$ & $\begin{array}{c}34 \\
33 \\
19 \\
6 \\
11 \\
11 \\
\mathbf{1 1 4}\end{array}$ & $\begin{array}{c}10 \\
10 \\
6 \\
2 \\
3 \\
3 \\
\mathbf{3 4}\end{array}$ & $\begin{array}{c}6 \\
3 \\
10 \\
0 \\
9 \\
6 \\
\mathbf{3 4}\end{array}$ & $\begin{array}{c}2 \\
1 \\
3 \\
0 \\
2 \\
2 \\
\mathbf{1 0}\end{array}$ & $\begin{array}{c}79 \\
78 \\
71 \\
35 \\
33 \\
40 \\
\mathbf{3 3 6}\end{array}$ & $\begin{array}{c}23,5 \\
23,2 \\
21,1 \\
10,7 \\
10 \\
12 \\
100\end{array}$ \\
\hline $\begin{array}{l}\text { ÍNDICE DE HIGIENE OR } \\
\text { Buena higiene } \\
\text { Regular higiene } \\
\text { Mala higiene } \\
\text { No aplica } \\
\text { Total }\end{array}$ & $\begin{array}{c}\text { PLAC } \\
2 \\
1 \\
0 \\
21 \\
\mathbf{2 4}\end{array}$ & $\begin{array}{l}1 \\
1 \\
0 \\
6 \\
7\end{array}$ & $\begin{array}{c}84 \\
44 \\
4 \\
32 \\
\mathbf{1 6 4}\end{array}$ & $\begin{array}{c}26 \\
12 \\
1 \\
10 \\
49\end{array}$ & $\begin{array}{c}46 \\
13 \\
3 \\
52 \\
\mathbf{1 1 4}\end{array}$ & $\begin{array}{c}13 \\
4 \\
1 \\
16 \\
\mathbf{3 4}\end{array}$ & $\begin{array}{c}12 \\
0 \\
0 \\
22 \\
\mathbf{3 4}\end{array}$ & $\begin{array}{c}3 \\
0 \\
0 \\
6 \\
10\end{array}$ & $\begin{array}{c}144 \\
58 \\
7 \\
127 \\
336\end{array}$ & $\begin{array}{c}43 \\
16 \\
4 \\
38 \\
\mathbf{1 0 0}\end{array}$ \\
\hline $\begin{array}{l}\text { ÍNDICE SALUD PERIODC } \\
\text { Normal } \\
\text { Gingivitis leve } \\
\text { Gingivitis moderada } \\
\text { Gingivitis severa } \\
\text { No aplica } \\
\text { Total }\end{array}$ & $\begin{array}{l}\mathbf{A L} \\
2 \\
0 \\
0 \\
1 \\
21 \\
\mathbf{2 4}\end{array}$ & $\begin{array}{l}1 \\
0 \\
0 \\
1 \\
6 \\
7\end{array}$ & $\begin{array}{c}28 \\
26 \\
27 \\
51 \\
32 \\
\mathbf{1 6 4}\end{array}$ & $\begin{array}{c}8 \\
8 \\
8 \\
15 \\
10 \\
49\end{array}$ & $\begin{array}{c}16 \\
17 \\
14 \\
16 \\
51 \\
\mathbf{1 1 4}\end{array}$ & $\begin{array}{c}4 \\
5 \\
4 \\
5 \\
15 \\
\mathbf{3 4}\end{array}$ & $\begin{array}{c}6 \\
4 \\
1 \\
0 \\
23 \\
\mathbf{3 4}\end{array}$ & $\begin{array}{c}2 \\
1 \\
1 \\
0 \\
7 \\
\mathbf{1 0}\end{array}$ & $\begin{array}{c}52 \\
47 \\
42 \\
68 \\
127 \\
\mathbf{3 3 6}\end{array}$ & $\begin{array}{c}15 \\
14 \\
13 \\
20 \\
38 \\
\mathbf{1 0 0}\end{array}$ \\
\hline $\begin{array}{l}\text { ÍNDICE COP } \\
\text { Muy bajo } \\
\text { Bajo } \\
\text { Intermedio } \\
\text { Alto } \\
\text { Muy alto } \\
\text { Total }\end{array}$ & $\begin{array}{c}22 \\
0 \\
0 \\
0 \\
2 \\
\mathbf{2 4}\end{array}$ & $\begin{array}{l}7 \\
0 \\
0 \\
0 \\
1 \\
7\end{array}$ & $\begin{array}{c}4 \\
1 \\
4 \\
2 \\
153 \\
\mathbf{1 6 4}\end{array}$ & $\begin{array}{c}1 \\
1 \\
1 \\
04 \\
45 \\
49\end{array}$ & $\begin{array}{c}53 \\
0 \\
2 \\
2 \\
57 \\
\mathbf{1 1 4}\end{array}$ & $\begin{array}{c}16 \\
0 \\
1 \\
0.4 \\
16 \\
\mathbf{3 4}\end{array}$ & $\begin{array}{c}27 \\
0 \\
0 \\
1 \\
6 \\
\mathbf{3 4}\end{array}$ & $\begin{array}{c}8 \\
0 \\
0 \\
0.2 \\
2 \\
\mathbf{1 0}\end{array}$ & $\begin{array}{c}106 \\
1 \\
6 \\
5 \\
218 \\
\mathbf{3 3 6}\end{array}$ & $\begin{array}{c}32 \\
1 \\
2 \\
1 \\
64 \\
\mathbf{1 0 0}\end{array}$ \\
\hline $\begin{array}{l}\text { DIAGNÓSTICO ESTOMA } \\
\text { Sano } \\
\text { Lengua saburral } \\
\text { Lengua fisurada } \\
\text { Perdida dimensión vertical } \\
\text { Otros } \\
\text { Total }\end{array}$ & $\begin{array}{c}\text { ÓGIC } \\
1 \\
12 \\
1 \\
9 \\
1 \\
\mathbf{2 4}\end{array}$ & $\begin{array}{l}1 \\
3 \\
1 \\
3 \\
1 \\
7\end{array}$ & $\begin{array}{c}25 \\
49 \\
30 \\
38 \\
22 \\
\mathbf{1 6 4}\end{array}$ & $\begin{array}{c}7 \\
15 \\
8 \\
11 \\
6 \\
49\end{array}$ & $\begin{array}{c}15 \\
56 \\
19 \\
20 \\
4 \\
\mathbf{1 1 4}\end{array}$ & $\begin{array}{c}5 \\
17 \\
6 \\
6 \\
1 \\
\mathbf{3 4}\end{array}$ & $\begin{array}{c}12 \\
13 \\
4 \\
5 \\
0 \\
\mathbf{3 4}\end{array}$ & $\begin{array}{c}3 \\
4 \\
1 \\
1 \\
0 \\
\mathbf{1 0}\end{array}$ & $\begin{array}{c}53 \\
130 \\
54 \\
72 \\
27 \\
\mathbf{3 3 6}\end{array}$ & $\begin{array}{c}16 \\
39 \\
16 \\
21 \\
8 \\
\mathbf{1 0 0}\end{array}$ \\
\hline $\begin{array}{l}\text { ALBÚMINA } \\
\text { Normal } \\
\text { Alta } \\
\text { Baja } \\
\text { Sin examen } \\
\text { Total }\end{array}$ & $\begin{array}{c}21 \\
0 \\
2 \\
1 \\
\mathbf{2 4}\end{array}$ & $\begin{array}{c}6 \\
0 \\
1 \\
0.5 \\
7\end{array}$ & $\begin{array}{c}138 \\
0 \\
22 \\
4 \\
\mathbf{1 6 4}\end{array}$ & $\begin{array}{c}41 \\
0 \\
7 \\
1 \\
49\end{array}$ & $\begin{array}{c}61 \\
0 \\
46 \\
7 \\
114\end{array}$ & $\begin{array}{c}18 \\
0 \\
14 \\
2 \\
\mathbf{3 4}\end{array}$ & $\begin{array}{c}31 \\
1 \\
0 \\
2 \\
\mathbf{3 4}\end{array}$ & $\begin{array}{c}9 \\
1 \\
0 \\
0.5 \\
\mathbf{1 0}\end{array}$ & $\begin{array}{c}251 \\
1 \\
70 \\
14 \\
\mathbf{3 3 6}\end{array}$ & $\begin{array}{c}74 \\
1 \\
21 \\
4 \\
\mathbf{1 0 0}\end{array}$ \\
\hline $\begin{array}{l}\text { TRANSFERRINA } \\
\text { Normal } \\
\text { Alta } \\
\text { Baja } \\
\text { Sin examen } \\
\text { Total }\end{array}$ & $\begin{array}{c}3 \\
0 \\
1 \\
20 \\
\mathbf{2 4}\end{array}$ & $\begin{array}{c}1 \\
0 \\
0.3 \\
6 \\
7\end{array}$ & $\begin{array}{c}109 \\
4 \\
44 \\
7 \\
\mathbf{1 6 4}\end{array}$ & $\begin{array}{c}32 \\
1 \\
13 \\
2 \\
49\end{array}$ & $\begin{array}{c}48 \\
3 \\
13 \\
50 \\
\mathbf{1 1 4}\end{array}$ & $\begin{array}{c}14 \\
0.8 \\
4 \\
15 \\
\mathbf{3 4}\end{array}$ & $\begin{array}{c}2 \\
1 \\
2 \\
29 \\
\mathbf{3 4}\end{array}$ & $\begin{array}{c}1 \\
0.2 \\
0.7 \\
9 \\
\mathbf{1 0}\end{array}$ & $\begin{array}{c}162 \\
8 \\
60 \\
106 \\
336\end{array}$ & $\begin{array}{c}48 \\
2 \\
18 \\
32 \\
\mathbf{1 0 0}\end{array}$ \\
\hline $\begin{array}{l}\text { FERRITINA } \\
\text { Normal } \\
\text { Alta } \\
\text { Baja } \\
\text { Sin examen } \\
\text { Total }\end{array}$ & $\begin{array}{c}13 \\
6 \\
0 \\
5 \\
\mathbf{2 4}\end{array}$ & $\begin{array}{l}4 \\
2 \\
0 \\
1 \\
7\end{array}$ & $\begin{array}{c}79 \\
80 \\
0 \\
5 \\
\mathbf{1 6 4}\end{array}$ & $\begin{array}{c}23 \\
24 \\
0 \\
1 \\
49\end{array}$ & $\begin{array}{c}33 \\
73 \\
2 \\
6 \\
114\end{array}$ & $\begin{array}{c}10 \\
22 \\
0.5 \\
1 \\
\mathbf{3 4}\end{array}$ & $\begin{array}{c}2 \\
4 \\
1 \\
27 \\
\mathbf{3 4}\end{array}$ & $\begin{array}{c}1 \\
1 \\
0.5 \\
9 \\
\mathbf{1 0}\end{array}$ & $\begin{array}{c}127 \\
163 \\
3 \\
43 \\
\mathbf{3 3 6}\end{array}$ & $\begin{array}{c}38 \\
49 \\
1 \\
12 \\
100\end{array}$ \\
\hline
\end{tabular}

(4.16\%) pacientes en prediálisis, 44 de 164 (26.82\%) en hemodiálisis, 13 de 114 (11.40\%) en diálisis peritoneal y dos de 34 (5.88\%) postrasplante renal. Bajos valores de albúmina (menor a 3.0) se identificaron dos (8.3\%) en terapia predialítica, $22(13.41 \%)$ en hemodiálisis, $46(40.35 \%)$ en diálisis peritoneal, y $0(0 \%)$ en postrasplante renal. Ferritina mayor a $500 \mathrm{mg} / \mathrm{dL}$ se encontró en seis $(25 \%)$ de los pacientes en prediálisis, 80 (48.7\%) en hemodiálisis, 73 (64.03\%) en diálisis peritoneal y cuatro $(11.76 \%)$ postrasplante renal (Tabla 2).
Sólo cinco pacientes cumplieron con el criterio de la presencia simultánea de saturación de transferrina menor a $20 \%$, albúmina menor a 3.0 y ferritina mayor a 500 , con lo cual tratar de establecer correlación entre salud periodontal y estado inflamatorio era estadísticamente improbable.

Se usó eritropoyetina en 222 pacientes con un promedio de dosis utilizada de 7300 unidades/semana. En el grupo de pacientes que recibieron eritropoyetina 111 de 222 requirieron dosis mayores de 6000 unidades/semana (dosis alta), y en ellos los índices se aplicaron a 82 pacientes dentados 
obteniéndose los siguientes resultados: $56(67 \%)$ presentaron buena higiene oral, $22(27 \%)$ regular higiene oral y cuatro (6\%) mala higiene oral. En salud periodontal 19 (23.52\%) pacientes presentaron encía sana, 19 (23.52\%) gingivitis leve, 14 (17.64\%) gingivitis moderada y 30 (35.2\%) gingivitis severa. No se encontró concordancia entre dosis altas de eritropoyetina y severidad de la gingivitis.

De los 82 pacientes dentados que necesitaron más de 6000 unidades de eritropoyetina a la semana, $25(30.4 \%)$ pacientes tuvieron baja saturación de transferrina, 14 (17.07\%) albúmina baja y 44 (53.66\%) ferritina alta. Presencia simultánea de las tres variables de inflamación sólo se detectó en un paciente, no siendo posible establecer algún tipo de correlación estadística en inflamación y dosis de eritropoyetina requerida.

\section{Discusión}

La salud oral en cualquier grupo de población refleja la interacción entre hábitos de higiene oral y mecanismos de defensa de la cavidad oral entre los cuales se identifican la integridad de mucosa y dientes, descamación celular, masticación, deglución y succión, tejidos linfoides, saliva, líquido crevicular (gingival) y remoción mecánica de detritos celulares en la superficie, ello con el fin de evitar la colonización y sobrecrecimiento microbiano (11). Entre estos últimos la saliva que posee un $\mathrm{PH}$ neutro tiene importantes habilidades protectoras manteniendo la integridad de la mucosa oral y de los dientes, de ahí que la reducción en la tasa de flujo salival de lugar a xerostomía, apariencia atrófica de la mucosa oral e incremento en el riesgo de caries $(12,13)$.

Varios factores pueden favorecer la aparición de enfermedad periodontal en pacientes con ERC: malos hábitos de higiene oral, disminución en la tasa de flujo salival, cambios en el PH salival, inmunosupresión crónica, disminución en la curación de heridas, destrucción alveolar por osteodistrofia renal, diátesis hemorrágica y desnutrición $(1,14,15)$.

En relación con los hábitos de higiene oral en un estudio en el sureste de Virginia se evaluaron 45 pacientes con ERC en terapia hemodialítica, detectándose restos alimenticios en el $100 \%$ de ellos, reflejándose en esa región malos hábitos de salud oral (14).

Disminución en la tasa de flujo salival ha sido demostrada principalmente en pacientes en terapia hemodialítica, ello podría ser consecuencia de la restricción en la toma de líquidos que frecuentemente se les recomienda (16-18); sin embargo, para otros autores ello puede ser consecuencia de afectación directa de la glándulas salivales por el medio urémico $(19,20)$. En un estudio reciente (21) se observó que la reducción en la tasa de flujo salival en pacientes en diálisis peritoneal aumentó el riesgo de caries.

Valores altos de PH salival se han observado en pacientes con ERC (18), ello podría ser el resultado de altas concentraciones salivares de amonio, las que derivan de la hidrólisis de la urea, principalmente en pacientes subdializados. El amonio por ser un captador de hidrogeniones eleva el PH salival $(22,23)$.
La inmunodeficiencia ha sido demostrada ampliamente en pacientes con enfermedad renal crónica, con disturbios en la inmunidad celular y humoral predisponiendo a infecciones crónicas como la periodontitis $(24,25)$. Gavaldá y cols. examinaron la mucosa oral de individuos con falla renal crónica y notaron lesiones severas en la mucosa, estomatitis urémica e infección por cándida en $37 \%$ de los pacientes. La alta prevalencia de candidiasis oral detectada podría ser explicable por el estado de inmunodeficiencia que acompaña a la ERC (26).

Los pacientes en hemodiálisis están expuestos a repetitivas anticoagulaciones sistémicas debido a la utilización de altas dosis de heparina durante el procedimiento extracorpóreo, la anticoagulación predispone a sangrado gingival, lo que podría facilitar la colonización bacteriana y su posterior crecimiento con propagación de la enfermedad periodontal (27).

La desnutrición por afectar la inmunidad, y alteraciones en el metabolismo óseo y mineral al afectar el balance calcio-fósforo y mineralización podrían también alterar la superficie ósea periodontal y favoreciendo la aparición de enfermedad periodontal.

Gran cantidad de estudios han evaluado la prevalencia de enfermedad periodontal en pacientes con ERC, encontrándose resultados variables, algunos describen alta prevalencia de enfermedad periodontal y factores que predisponen a su aparición y progresión $(1,26,28-30)$; por el contrario, en otros en los cuales se compara con la población normal la prevalencia no es diferente (31-33). Se puede concluir por lo tanto que en la aparición de enfermedad periodontal podrían intervenir diversas variables: etnicidad, diferencias geográficas, atención médica dental, estado cultural, socioeconómico, raza, edad y enfermedad de base.

Las implicaciones de la presencia de enfermedad periodontal es su capacidad de generar un estado inflamatorio sistémico que predispone a enfermedades cardiovasculares, marcadores del mismo han sido detectados, y de ellos la proteína $\mathrm{C}$ reactiva (PCR) se ha encontrado elevada en diversos estudios (34-37). Análisis de datos del NHANES III demostraron asociación positiva entre la severidad de la enfermedad periodontal y valores de PCR (38), lo que se confirmó en el estudio Life de New York (36). Asociación directa entre enfermedad periodontal y enfermedad coronaria ha sido demostrada recientemente en dos metaanálisis $(39,40)$. En el estudio de Bahekar y colaboradores en el que se incluyeron 86092 pacientes se concluyó que la periodontitis aumentaba en 1.14 veces el riesgo coronario (40) .

En nuestro estudio encontramos que la población de pacientes postrasplante renal en general demostró una buena salud oral, lo cual refleja la atención y exigencia previa al trasplante por el grupo de odontología. El grupo de pacientes en prediálisis que pudo ser evaluable fue muy pequeño, y en términos generales afectó poco los resultados. El grupo de pacientes en programa de hemodiálisis y diálisis peritoneal sí alcanzó un número significativo sobre el cual fue posible el análisis estadístico. 
De 336 pacientes estudiados 127 (38\%) presentaban prótesis total o eran edentados. En los 209 pacientes a quienes se pudo evaluar, la mayoría de ellos se encontraba en terapia de hemodiálisis y diálisis peritoneal, los resultados de higiene oral se encontraron así: buena en 63-68\%, regular en $20-33 \%$ y mala en 3-5\%. La comparación de estos resultados con otros reportes internacionales $(1,26,28-30)$, arroja diferencias importantes, pues la mayoría demuestran una mala higiene oral en diferentes grupos de tratamientos de la ERC, lo que refleja la influencia de variables geográficas y culturales.

En relación con la enfermedad periodontal, principalmente en los grupos de pacientes en hemodiálisis y diálisis peritoneal, estaba presente entre $74-78 \%$ de los pacientes, siendo leve en $19-27 \%$, moderada en $20-22 \%$ y severa en $36-38 \%$. Estos datos son llamativos y sugieren que el medio urémico, aun en presencia de buenos hábitos de salud oral, es un factor que predispone a la enfermedad periodontal independientemente del tipo de terapia dialítica.

El índice COP-D encontrado, con predominio de pacientes en los dos extremos muy bajo (32\%) y muy alto (64\%) nos lleva a inferir que hay una alta prevalencia de caries, dientes obturados y perdidos que coincide a nivel internacional con estudios como el de Gavaldá y cols. que indican una mayor prevalencia de caries entre los pacientes adultos con ERC en hemodiálisis que en los controles sanos (26).

En el diagnóstico estomatológico 39\% de los pacientes con lengua saburral, $16 \%$ con lengua fisurada y $6.5 \%$ con candidiasis, en presencia de buena a regular higiene oral indica sobrecrecimiento bacteriano anormal en las superficies de tejidos blandos, siendo favorecido por el medio urémico y disminución en la secreción salival, con deficiencia en la autoclisis o remoción de detritos de la cavidad oral y sobreinfección por cándida.

Se ha sugerido que la inflamación crónica puede dar lugar a alteraciones en la síntesis de proteínas inflamatorias, elevándose la ferritina y disminuyéndose los niveles de albúmina y transferrina, ello no fue confirmado en nuestro estudio, muy probablemente por la intervención precoz y acertada sobre cada una de las alteraciones de laboratorio que se presentan en nuestros pacientes por parte de los nefrólogos y grupo de apoyo. Recientemente la utilización de un grupo más sensible de marcadores inflamatorios entre los que se incluyó PCR altamente sensible, IL-1B, IL-6, IL-8, TNF-alfa y homocisteína detectó asociación sinérgica entre periodontitis y síndrome metabólico coexistente, lo cual nos lleva a recomendar la necesidad de utilizar en estudios futuros este tipo de marcadores (41). En nuestro estudio tampoco se detectó correlación entre la dosis de eritropoyetina y los marcadores inflamatorios, con lo cual se establece que otras variables pueden ser más importantes en determinar sus requerimientos.

En conclusión la enfermedad periodontal es común en la ERC, a pesar de buenos hábitos de higiene oral, lo cual sugiere que el medio urémico y la baja tasa de flujo salival en estos pacientes puede predisponer a su aparición. Identificar la enfermedad periodontal en pacientes con ERC es primordial por sus implicaciones pronósticas, no sólo locales sino sistémicas principalmente cardiovasculares, predisponiéndolos a alta morbimortalidad. Intervenciones que corrijan los factores desencadenantes deben ser llevadas a cabo en forma prioritaria. El uso de goma de mascar sin azúcar o colutorios desinfectantes sin alcohol podrían ayudar a remover la placa dental bacteriana o biofilm en dientes y lengua contribuyendo a mejorar la salud oral (42). Alterar el contenido bacterial subgingival, desbridamientos mecánicos de cálculos y placas subgingivales, eliminación quirúrgica de bolsas periodontales, son otras alternativas, pero es importantísima la estrecha comunicación entre el odontólogo del paciente y el nefrólogo, con el fin de optimizar el manejo periodontal. Adecuada profilaxis y tratamiento de la enfermedad periodontal deben iniciarse precozmente y en tempranos estadios de la enfermedad renal crónica.

\section{Sugerencias de manejo en salud oral de pacientes con ERC}

- Los pacientes con ERC deben recibir valoración odontológica al menos un mes antes de iniciar su primera terapia de diálisis. Esta valoración es esencial para eliminar los focos infecciosos potenciales de la cavidad oral.

- Todos los pacientes en diálisis deben recibir valoración y control cuatro veces al año.

- Se debe incorporar un programa de mantenimiento de salud oral en pacientes que reciben diálisis dentro del grupo multidisciplinario que atiende este grupo de pacientes.

- Profilaxis antibiótica previa a procedimientos en cavidad oral invasivos, que incluyen raspaje radicular y exodoncias habilita a los pacientes a tolerar en mejor forma el tratamiento dental.

- Las citas odontológicas deben ser realizadas el día después de la hemodiálisis para evitar el riesgo de sangrado.

- Debido a la alta incidencia de hipertensión en pacientes con ERC, usar con cuidado anestésico que contenga vasoconstrictores.

- Procedimientos periodontales que involucran hueso así como los injertos o implantes dentales están contraindicados en pacientes con osteodistrofia renal significativa y en aquellos que reciben bifosfonatos por el riesgo de osteonecrosis maxilar (43).

\section{Agradecimientos}

A la Dra. María Fernanda Navia Jutchenko por su colaboración en la valoración de los pacientes en hemodiálisis.

A los estudiantes de la Universidad Autónoma en rotación hospitalaria por el Hospital Infantil Universitario y el Hospital de Caldas durante los periodos 2009 y 2010, por su participación en el proceso evaluativo de los pacientes.

A los funcionarios de la Unidad RTS sucursal Caldas, por su apoyo en la detección de los pacientes. 


\section{Referencias}

1. Borawski J, Wilczynska-Borawska M, Stokowska W, Mysliwiec M. The periodontal status of pre-dialysis chronic kidney disease and maintenance dialysis patients. Nephrol Dial Transplant 2007; 22: 457-64.

2. Tonetti M S, D'Aiuto F, Nibali L, Donald A, Storry C, Parkar M, Suvan J, Hingorani A D, Vallance P, Deanfield $\mathbf{J}$. Treatment of periodontitis and endothelial function. $N$ Eng J Med 2007; 356: 911-20.

3. III Estudio Nacional de Salud Bucal - ENSAB III, II Estudio Nacional de factores de riesgo de enfermedades crónicas - ENFREC II. República de Colombia. Ministerio de salud 1999; VII: 19-147.

4. De Mutsert R, Grootendorst DC, Indemans F, Boeschoten EW, Krediet RT, Dekker FW. Association between serum albumin and mortality in dialysis patients is partly explained by inflammation, and not by malnutrition. J Ren Nutr 2009; 19:127-35.

5. Fox E R, Benjamin E J, Sarpong D F, Nagarajarao H, Taylor J K, Steffes M W, Salahudeen A K, Flessner M F, Akylbekova E L, Fox C S, Garrison $\mathbf{R} \mathbf{J}$, Taylor $\mathbf{H} \mathbf{A}$. The relation of C-reactive protein to chronic kidney disease in African americans: the Jackson Heart Study. BMC Nephrology 2010; 11: 1-7.

6. Ikizler TA, Wingard RL, Harvell J, Shyr Y, Hakim RM: Association of morbidity with markers of nutrition and inflammation in chronic hemodialysis patients: a prospective study. Kidney Int 1999; 55:1945-51.

7. Myron J A, Merlini G, Sheldon J, Ichihara K. Clinical indications for plasma protein assays: transthyretin (prealbumin) in inflammation and malnutrition. Clin Chem Lab Med 2007; 45: 419-26.

8. Loe H, Silness J. Periodontal Disease in pregnancy. Acta Odont Scand 1963; 21: 533-8.

9. Silness J, Loe H. Periodontal disease in pregnancy. Acta Odontol Scand 1964; 22: $121-8$

10. Organización Mundial de la Salud. Investigaciones de Salud Oral Básica: Métodos Básicos. Ginebra 1987

11. Negroni M. (2009). Microbiología estomatológica. Fundamentos y guía práctica. Editorial Médica Panamericana S.A. Buenos Aires, Argentina.

12. Mandel ID. The functions of saliva. J Dent Res 1987; 66: 623-7.

13. Vissink A, Panders AK, Gravenmade E, Vermey A. The causes and consequences of hyposalivation. Ear Nose Throat J 1988; 67: 166-76.

14. Naugle K, Darby ML, Bauman DB, Lineberger LT, Powers R. The Oral Health Status of individuals on renal dialysis. Ann Periodontol 1998; 3: 197-205.

15. Kramer H, Toto R, Peshock R, Cooper R, Victor R. Association between Chronic Kidney Disease and Coronary Artery Calcification: The Dallas Heart Study. J Am Soc Nephrol 2005; 16: 507-13.

16. Epstein SR, Mandel I, Scopp IW. Salivary composition and calculus formation in patients undergoing hemodialysis. J Periodontol 1980; 51: 336-8.

17. Martins C, Siqueira WL, de Oliveira E. Salivary analysis of patients with chronic renal failure undergoing hemodialysis. Spec Care Dentist 2006; 26: 205-8.

18. Bayraktar G, Kazancioglu R, Bozfakioglu S, Yildiz A, Ark E. Evaluation of salivary parameters and dental status in adult hemodialysis patients. Clin Nephrol 2004; 62: 380-3

19. Kao C H, HsiehJ F, Tsai S C, Ho Y J, Chang H R. Decreased salivary function in patients with end-stage renal disease requiring hemodialysis. Am J Kidney Dis 2000; 36: 1110-4.

20. Postorino M, Catalano C, Martorano C, Cutrupi S, Marino C, Cozzupoli P, Scudo P, Zoccali C. Salivary and lacrimal secretion is reduced in patients with ESRD. Am J Kidney Dis 2003; 42: 722-8.

21. Bayraktar G, Kurtulus I, Kazancioglu R, Bayramgurler I, Cintan S, Bural C, Bozfakioglu S, Issever H, Yildiz A. Oral health and inflammation in patients with end-stage renal failure. Perit Dial Int 2009; 29: 472-9.

22. Al-Nowaiser A, Roberts G J, Trompeter R S, Wilson M, Lucas V S. Oral health in children with chronic renal failure. Pediatr Nephrol 2003; 18: 39-45.

23. Peterson S, Woodhead J, Crall J. Caries resistance in children with chronic renal failure: plaque $\mathrm{pH}$, salivary $\mathrm{pH}$, and salivary composition. Pediatr. Res 1985; 19: 796-9.

24. Chatenoud L, Herbelin A, Beaurain G. Immune deficiency of the uremic patient. Adv Nephrol Necker Hosp 1990; 19: 259-74.

25. Girndt M, Sester M, Sester U. Molecular aspects of T and B cell function in uremia. Kidney Int Suppl 2001; 78: S206-11.

26. Gavaldá C, Bagán J V, Scully C, Silvestre F J, Milián M A, Jiménez Y. Renal hemodialysis patients: oral, salivary, dental and periodontal findings in 105 adult cases. Oral Surg Oral Med Oral Pathol Oral Radiol Endod 1999; 5: 299-302.

27. Borawski J, Wilczyńska-Borawska M, Stokowska W, Myśliwiec M. The periodontal status of pre-dialysis chronic kidney disease and maintenance dialysis patients. Nephrol Dial Transplant 2007; 22: 457-64.

28. Bayraktar G, Kurtulus I, Duraduryan A, Cintan S, Kazancioglu R, Yildiz A et al. Dental and periodontal findings in hemodialysis patients. Oral Diseases 2007; 13: 393-7.

29. Souza CR, Libério SA, Guerra RN, et al. Assessment of periodontal condition of kidney patients in hemodialysis. Rev Assoc Med Bras 2005; 51: 285-9.

30. Kshirsagar A V, Craig R G, Moss K L, Beck J D, Offenbacher S, Kotanko P, Klemmer P J, Yoshino M, Levin N W, Yip J K, Almas K, Lupovici E M, Usvyat L A, Falk R J. Periodontal disease adversely affects the survival of patients with end-stage renal disease. Kidney Int 2009; 75: 746-51.

31. Castillo A, Mesa F, Liébana J, García-Martinez O, Ruiz S, García-Valdecasas J, O>Valle F.Periodontal and oral microbiological status of an adult population undergoing haemodialysis: a cross-sectional study. Oral Diseases 2007; 13: 198205.

32. Bots C P, Poorterman J H G, Brand H S, Kalsbeek H, Van Amerongen B $\mathbf{M}$, Veerman $\mathbf{E} \mathbf{C} \mathbf{I}$. The oral health status of dentate patients with chronic renal failure undergoing dialysis therapy. Oral Diseases 2006;12: 176-80.

33. Marinho J S S, Carmona I T, Loureiro A, Posse J L, Caballero L G, Dios P D. Oral health status in patients with moderate-severe and terminal renal failure. Med Oral Patol Oral Cir Bucal 2007; 12: E305-10.

34. Beck JD, Slade G, Offenbacher S. Oral disease, cardiovascular disease and systemic inflammation. Periodontol 2000; 23: 110-20.

35. Loos B G, Craandijk J, Hoek F J, Paulien M E, Dillen W, Van Der Velden U. Elevation of Systemic Markers Related to Cardiovascular Diseases in the Peripheral Blood of Periodontitis Patients. J Periodontal 2000; 71: 1528-34.

36. Noack B, Genco R J, Trevisan M, Grossi S, Zambon J J, De Nardin E. Periodontal Infections Contribute to Elevated Systemic C-Reactive Protein Level. J Peridontal 2001; 72: 1221-7.

37. Mattila K, Vesanen M, Valtonen V, Nieminen M, Palosuo T, Rasi V, Asikainen S. Effect of treating periodontitis on C-reactive protein levels: a pilot study. $B M C$ Infect Dis 2002; 2: 30 .

38. Slade GD, Offenbacher S, Beck JD. Acute-phase inflammatory response to periodontal disease in the US population. J Dent Res 2000; 79: 49-57.

39. Humphrey LL, Fu R, Buckley DI, Freeman M, Helfand M. Periodontal disease and coronary heart disease incidence: a systematic review and meta-analysis. $J$ Gen Intern Med 2008; 23: 2079-86.

40. Bahekar AA, Singh S, Saha S, Molnar J, Arora R. The prevalence and incidence of coronary heart disease is significantly increased in periodontitis: a meta-analysis. Am Heart J 2007; 154: 830-7.

41. Han DH, Shin HS, Kim MS, Paek D, Kim HD. Group of serum inflammatory markers and periodontitis-metabolic syndrome coexistence en Koreans. J Periodontol 2011; posted online on August 26.

42. García E, S. Cruz Mérida S C, Mondragón Padilla A. Pérdida de dientes en pacientes diabéticos con y sin insuficiencia renal crónica y diálisis. Nefrología 2008; 28: 645-8.

43. Yarom N, Yahalom R, Shoshani Y, Hamed W, Regev E, Elad S. Osteonecrosi of the jaw induced by orally administered bisphosphonates: incidence, clinical features, predisposing factors and treatment outcome. Osteoporos Int 2007; 18: 1363-70. 